\title{
Observing the Responses of the Interface Between New Bioactive Glasses and Human Osteoblast Cells (HOCs) by TEM
}

\author{
Naho Akamatsu, Noriko Suzuki, Wataru Ofusa, Yukimi Suzuki, Hitoshi Oguchi* \\ Department of Geriatric Dentistry, Tsurumi University School of Dental Medicine, Yokohama City, Japan \\ Email address: \\ akamatsu-nh@tsurumi-u.ac.jp (N. Akamatsu),suzuki-n@tsurumi-u.ac.jp (N. Suzuki), Ofusa-w@tsurumi-u.ac.jp (W. Ofusa), \\ suzuki-y@tsurumi-u.ac.jp (Y. Suzuki),oguchi-h@tsurumi-u.ac.jp (H. Oguchi) \\ ${ }^{*}$ Corresponding author
}

\section{To cite this article:}

Naho Akamatsu, Noriko Suzuki, Wataru Ofusa, Yukimi Suzuki, Hitoshi Oguchi. Observing the Responses of the Interface Between New Bioactive Glasses and Human Osteoblast Cells (HOCs) by TEM. International Journal of Dental Medicine. Vol. 4, No. 1, 2018, pp. 19-26. doi: $10.11648 / \mathrm{j} . \mathrm{ijdm} .20180401 .15$

Received: June 21, 2018; Accepted: July 9, 2018; Published: August 4, 2018

\begin{abstract}
The purpose of this study was to investigate differences in the interface between new bioactive glasses (RKKP, RBP1, RBP2) and mirror-polished titanium alloys in the evaluation of human osteoblast cells (HOCs) by TEM. Mirror-polished titanium alloy (MTi), bioactive glass (RKKP, RBP1, RBP2), and plastic culture dishes (Falcon [F]; used as a control) were used in this study. $1.0 \times 10^{5}$ HOCs were plated on each of the materials and cultured for 1 week. For TEM, HOCs were fixed in $2.5 \%$ glutaraldehyde, post-fixed in 1\% osmium tetroxide, dehydrated, and embedded in Epon 812 . After polymerization for 48 hours, the sample was removed with liquid nitrogen, and the specimen was re-embedded in Epon 812, sliced into 200-nm-thick semithin sections, and stained with a mixture of $1 \%$ toluidine blue, $1 \%$ Azur II, and 1\% borax. After confirming the presence of cells, the specimens were sliced at a thickness of approximately $78 \mathrm{~nm}$, double-stained with uranyl acetate and lead citrate, and examined under a TEM. As results, In Falcon, we found an extremer on the material side, focal contact, and close contact in parts of the plate. In M-Ti, we observed a non-structured homogeneous layer. In RBP1 and RBP2, an intervening layer with a gel like condition of approximately $100 \mathrm{~nm}$ in thickness was observed. In RKKP the collagen fibers bonded directly, indicating the presence of a bone matrix of HOCs at all sites. In conclusion, the interface between the different bioactive materials and the bio-inactive material showed completely different phenomena. Furthermore, even the same bioactive materials showed different patterns because of their different composition. From these results, we concluded that RKKP might be the best biomaterial because it bonded directly to human osteoblast cells without an intervening layer.
\end{abstract}

Keywords: Bioactive Bioglass, Human Osteoblast Cells, Interface

\section{Introduction}

Many biomaterials are currently used for dental implants, as filling biomaterials for bone defects and for the reconstruction of the jaw. These include hydroxyapatite [1], titanium alloy [2], zirconia [3] and bioglass [4]. A common bioactive behavior of glasses, which is observed independently of their specific composition, is the formation of a calcium phosphate-rich layer after soaking with simulated body fluids. Many authors have reported that amorphous calcium phosphates initially form then crystallize to hydroxycarbonate-apatites and carbonate-apatite, which are analogous to the substances present in bone [4].

In 1969, Prof. Hench first discovered bioactive glass $\left(\mathrm{Na}_{2} \mathrm{O}-\mathrm{CaO}-\mathrm{P}_{2} \mathrm{O}_{5}-\mathrm{SiO}_{2}\right)$ and named it $45 \mathrm{~S} 5$. He reported the results of in vitro biocompatibility studies and in vitro tests of the interfacial bonding of implants to bone in 1971 [4, 5]. Hench [5] reported that the mechanism for the development of this bond involves the production of an amorphous ion surface gel on the bioglass. This gel induces osteogenesis by a chemotactic of osteoblasts. Furthermore, the glasses were bonded to a layer of collagen fibrils produced at the interface 
by osteoblasts. The chemical bonding of the HA layer to collagen created a strongly bonded interface [6]. However, fragility was a problem. In 1990, Yamamuro and Kokubo developed A/W (apatite-wollastonite) bioactive glass [7]. The material contained $38 \%$ oxyapatite and fluoroapatite, $34 \%$ $\beta$-wollastonite $\left(\mathrm{CaO}-\mathrm{SiO}_{2}\right)$, and $28 \%$ residual glass. However, manufacturing was a problem due to the hardness. Recently, there have been various studies on AP40 and RKKP [8-14]. AP40 and RKKP exchange ions far slower than Hench's glass (such as 45S5). The difference between AP40 and RKKP is the presence of $\mathrm{La}$ and Ta. Small amounts of $\mathrm{La}_{2} \mathrm{O}_{3}$ and $\mathrm{Ta}_{2} \mathrm{O}_{5}$ (RKKP) were added to furnish a possible nucleus for the deposition of ions involved in bone formation. The slower exchange of RKKP promotes the formation of silicate chain networks, as well as stabilizes and-more importantly-increases the packing density of the molecular network. In vitro experiments have shown that the presence of these oxides can modify the surface properties of the glass and influence the protein absorption kinetics. In 2008, we used SEM and TEM to demonstrate - for the first time-that RKKP is most biocompatible with Human Gingival Epithelial Cells (HGE-15 cells) [14] Furthermore, Ravaglioli and Krajewski developed RBP1 and RBP2 based on AP40 [13]. They reported that RBP1 and RBP2 are less stable than RKKP. (Consequently, their liquidus temperatures, which indicate in some way the strength of molecular bonds in the molecular network of the glass, are lower.)

In addition, studies of the ionic release rates of RBP1 and RBP2 have shown that these two new types of glass exchange ions with physiological solution more slowly in comparison to AP40 and RKKP. A slower release produces smaller changes in terms of the ionic presence and the physicochemical variations around the implanted piece of bioactive glass, as well as a higher charged positive/negative double layer. $\mathrm{ZnO}$ was added to both RBP1 and RBP2. $\mathrm{ZnO}$ is known as a cicatrizant agent and $\mathrm{Zn}$ ions are useful for controlling the solubility of the glass system since they reinforces its structure. $\mathrm{Sr}$ is related to the hardness. $\mathrm{Nb}$ has resistance for many chemical materials and manufacture easily at low temperature. In RBP1 and RBP2, $\mathrm{Zn}^{2+}$ - which is much more active than $\mathrm{Ta}^{5+} / \mathrm{La}^{3+}$ in RKKP-acts as a moderator of the ionic leaching rate $[8,13]$. In 2014, we first proved the biological responses of the interface between new bioglasses (RKKP, RBP1, RBP2) and HGE cells using TEM. As a result, only RKKP was found to directly bond to the cells without an intervening layer. On the other hand, in RBP1 and 2, we observed a gel-like layer at the interface on TEM photographs [15].

The purpose of this study was to investigate differences in the interface between new types of bioactive glass (RKKP, $\mathrm{RBP} 1, \mathrm{RBP} 2$ ) and a mirror-polished titanium alloy, using human osteoblast cells (HOCs) by TEM.

\section{Materials and Methods}

Samples: Three types of bioactive glass (RKKP, RBP1 and RBP 2, Institute of Science and Technology on Ceramic Materials, Faenza, Italy) were studied. Both RBP1 (AP40 + $\left.0.5 \% \mathrm{ZnO}+1 \% \mathrm{SrCo}_{3}\right)$ and $\mathrm{RBP} 2(\mathrm{AP} 40+0.5 \% \mathrm{ZnO}+1 \%$ $\mathrm{Nb}_{2} \mathrm{O}_{5}$ ) are based on AP40. We also used mirror-polished titanium alloy Ti-6Al-4V (M-Ti, machined by diamond powder, Advance Co., Ltd.). Tissue culture dishes (Falcon ${ }^{\circledR}$ 3001 [F], Becton Dickinson Co., Ltd) were used as a control. Each sample was a rectangular parallel pipe of $1.0 \mathrm{~mm}$ in thickness and $10 \mathrm{~mm}$ in length and width. We prepared 5 pieces of each sample for this study.

Biological evaluation: $1.0 \times 10^{6}$ of normal HOCs (CCS-2538: BioWhittaker. MD USA) were plated on the materials and cultured for 1 week at $37^{\circ} \mathrm{C}$ with osteoblast basal medium (Cloetisx, Biowhttaker, Inc. USA) in 5\% $\mathrm{CO}_{2}$. For examination by TEM, the HOCs were fixed in $2.5 \%$ glutaraldehyde ( $0.1 \mathrm{M}$ cacodylate buffer, $\mathrm{pH} 7.4)$, postfixed in $1 \%$ osmium tetroxide, dehydrated, and embedded in Epon 812. After polymerization for 48 hours, the sample was removed with liquid nitrogen, and the specimen was re-embedded in Epon 812, sliced into 200-nm-thick semi-thin sections (Reichert Ultracut-E), and stained with a mixture of $1 \%$ toluidine blue, 1\% Azur II, and 1\% borax. After confirming the presence of cells, the specimens were sliced at a thickness of approximately $78 \mathrm{~nm}$, double-stained with uranyl acetate and lead citrate, and examined using a transmission electron microscope (1200EX [JEM]: 80KV).

\section{Results}

In Falcon, a thin, dense layer that represented some type of adhesive protein or muco-polysaccharide was observed next to the material surface. In other parts, focal contact and closed contact and extracellular matrix from the cells were observed (Figure 1). In M-Ti, we did not observe direct bonding to the cells. Similarly to Falcon, the thin, dense layer was observed next to the material surface on M-Ti. Furthermore, a clear amorphous layer of 100-300 nm in thickness was observed between the HOCs and M-Ti (Figure 2). In RBP1, the HOCs were bonded to RBP1 intervening the amorphous layer of approximately 500nm in thickness (Figure 3). In RBP2, the findings were basically the same as those observed in RBP1, with an amorphous intervening layer observed between HOC and RBP2. On the other hand, in RKKP showed completely different findings to RBP1, and RBP2 (Figure 4, 5). RKKP bonded directly to the collagen fibers of the HOCs at all sites (Figure 6). 

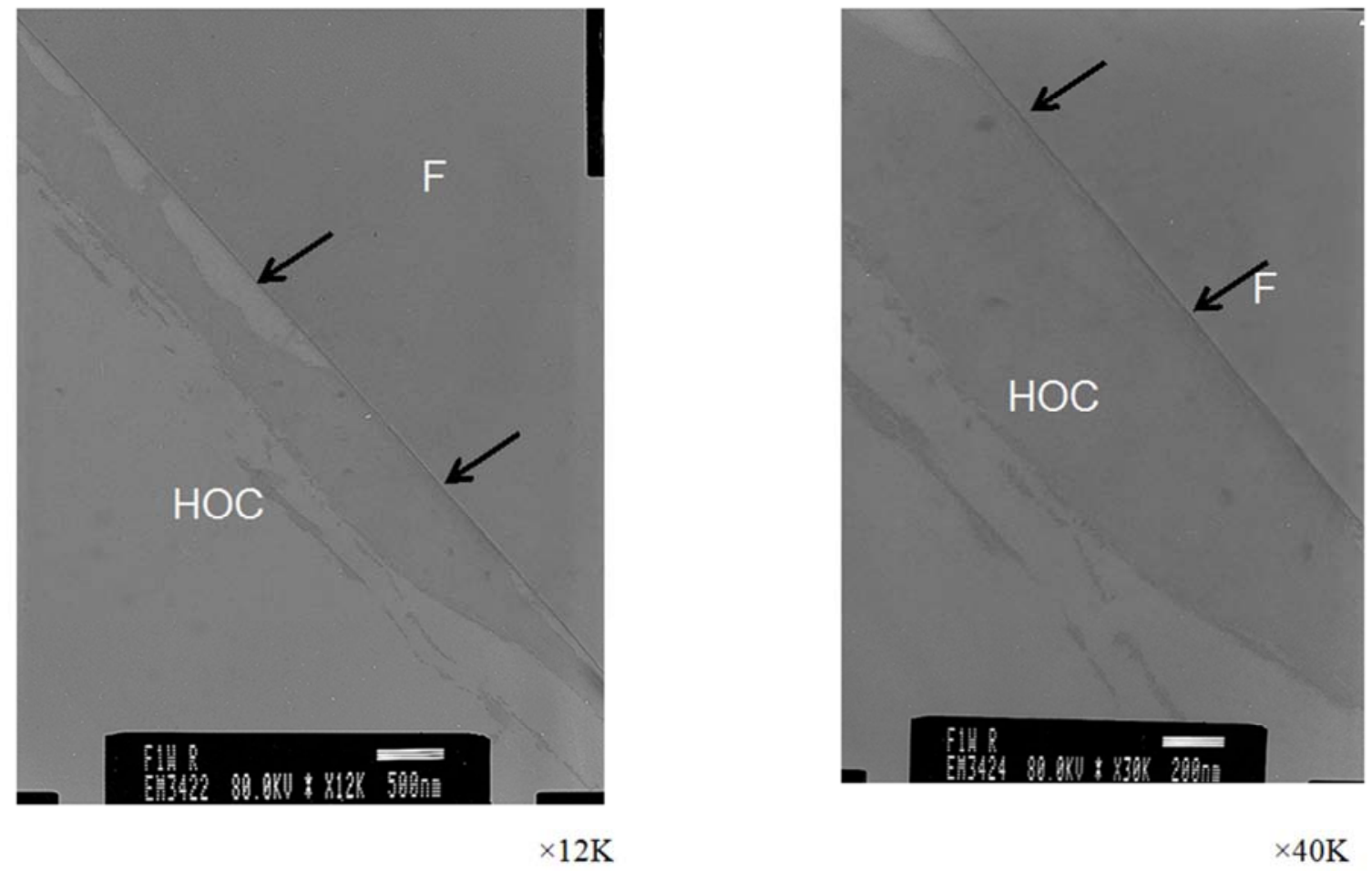

Plastic Culture dish (Falcon: 1W)

Figure 1. TEM photographs of Falcon 1 week after incubation. An extremely thin, dense layer was observed beside the material. In parts, focal contact, closed contact and extracellular matrix were seen.
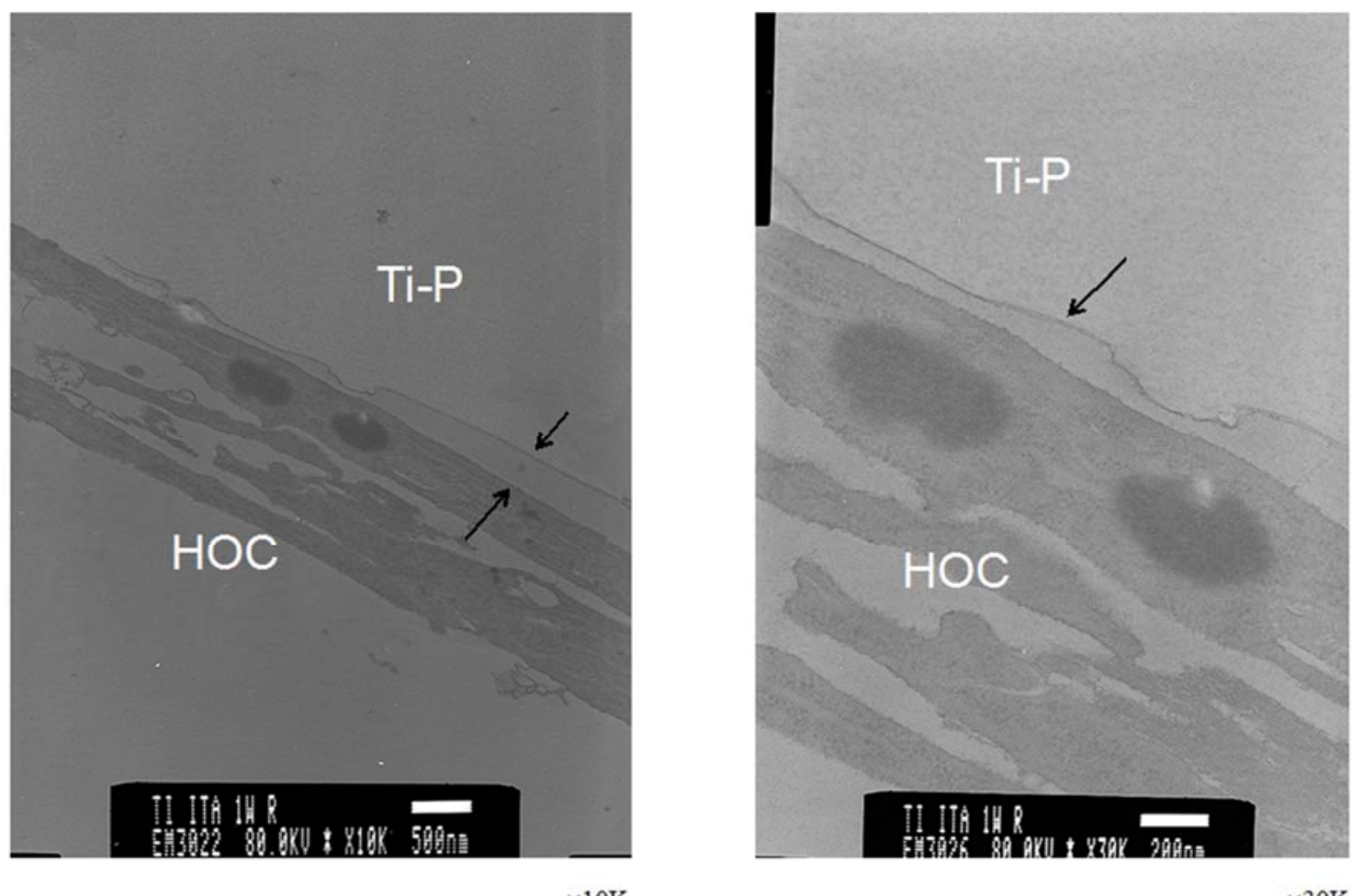

Titanium Plate (1 week)

Figure 2. TEM photographs of M-Ti 1 week after incubation. A clear amorphous layer (extracellular matrix) of 100-300 nm in thickness was seen between the cell membrane of the HOC and the thin, dense layer on the surface of M-Ti. 


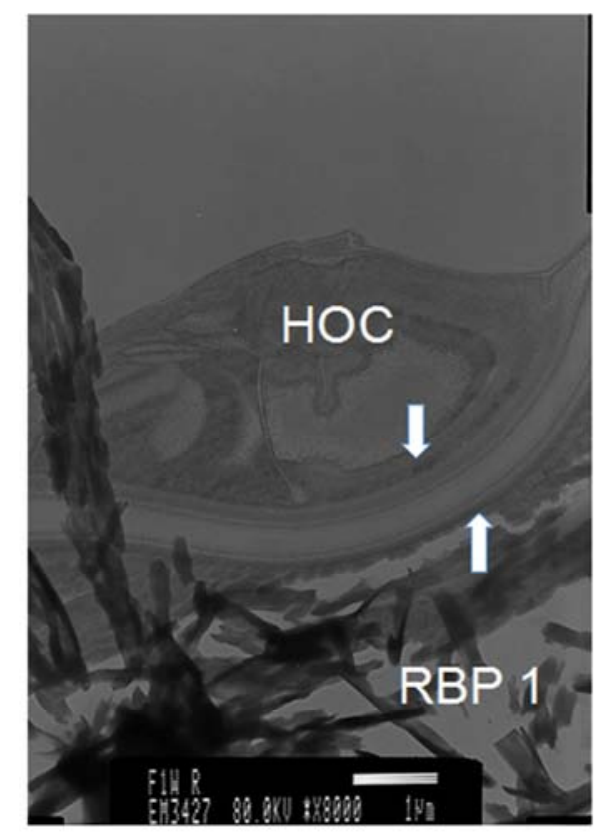

(a)

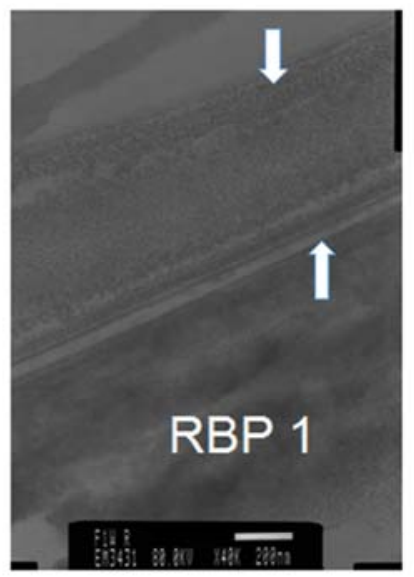

(b)

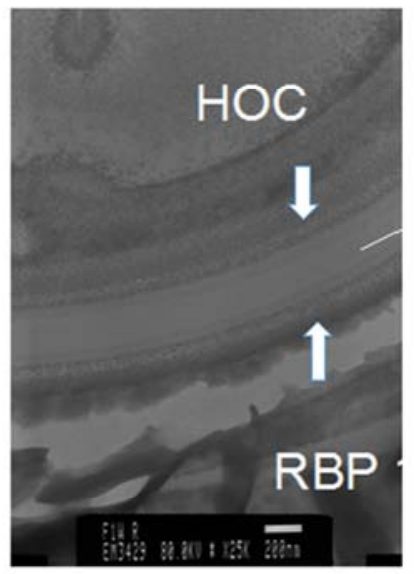

(c)

RBP1 (1 W)

Figure 3. TEM photographs of RBPI at 1 week after incubation. An intervening amorphous layer of approximately $500 \mathrm{~nm}$ in thickness was seen between HOC and RBP1. $(a: \times 8 K, b: \times 40 K, c: \times 25 K)$.

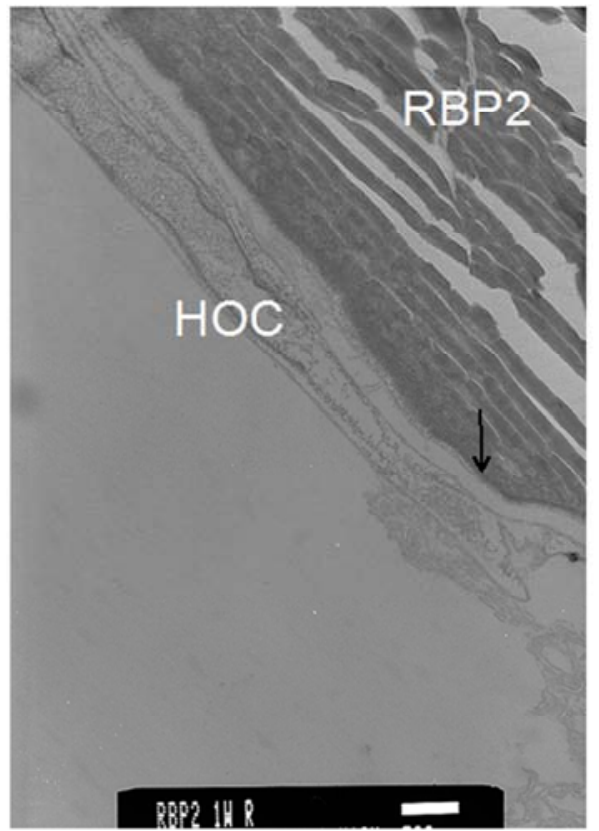

$\times 10 \mathrm{~K}$

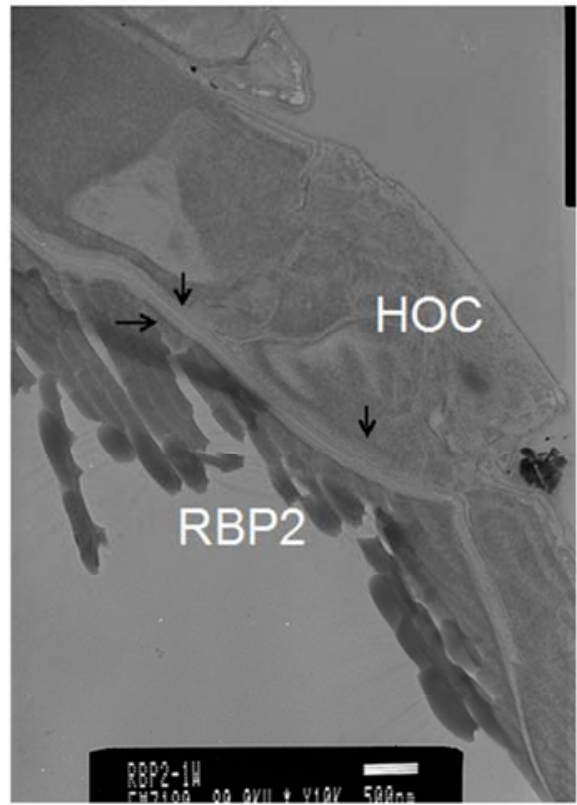

$\times 10 \mathrm{~K}$

RBP2 (1 W)

Figure 4. TEM photographs of RBP2 at 1 week after incubation. The findings were basically the same as those observed with RBP1. 


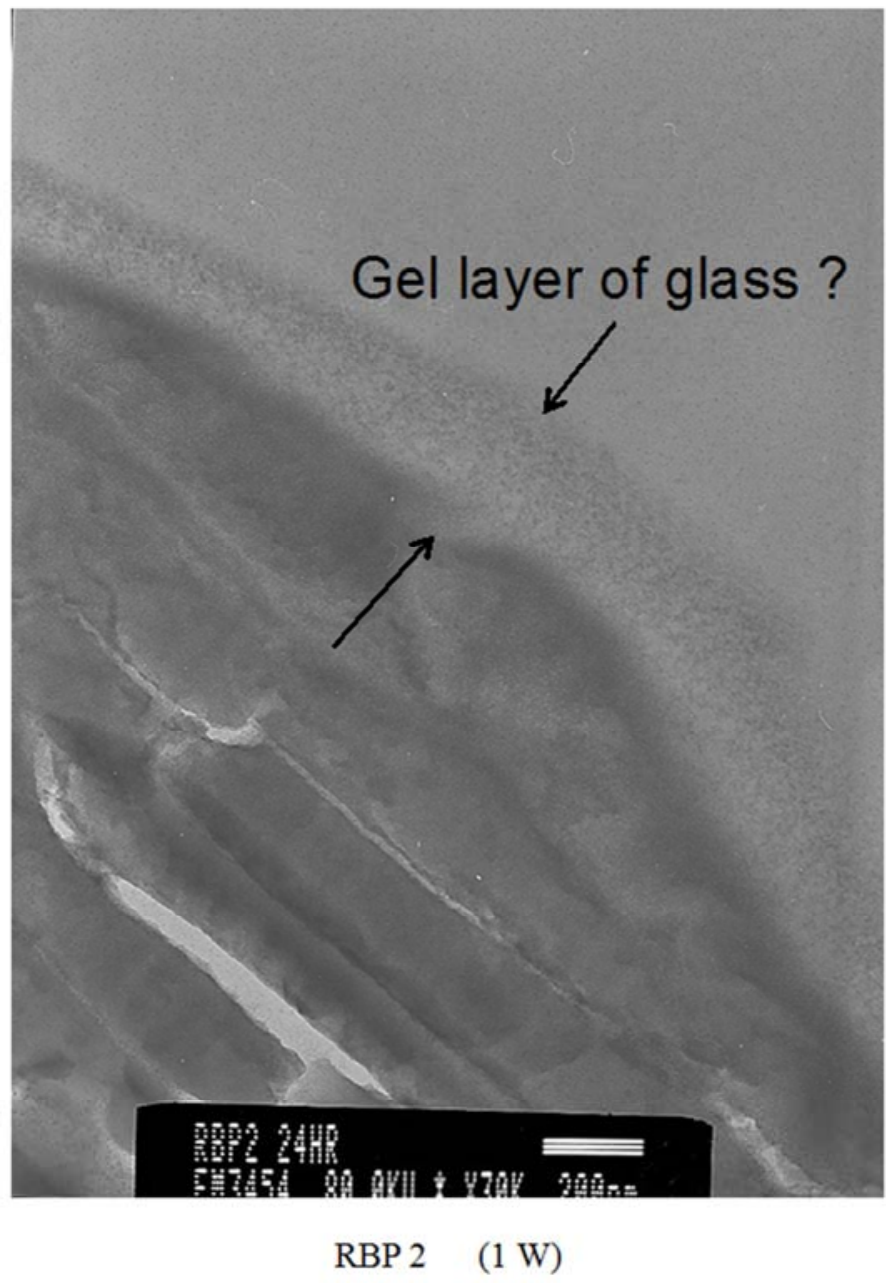

Figure 5. A gel layer of approximately $200 \mathrm{~nm}$ in thickness on the surface of RBP2.

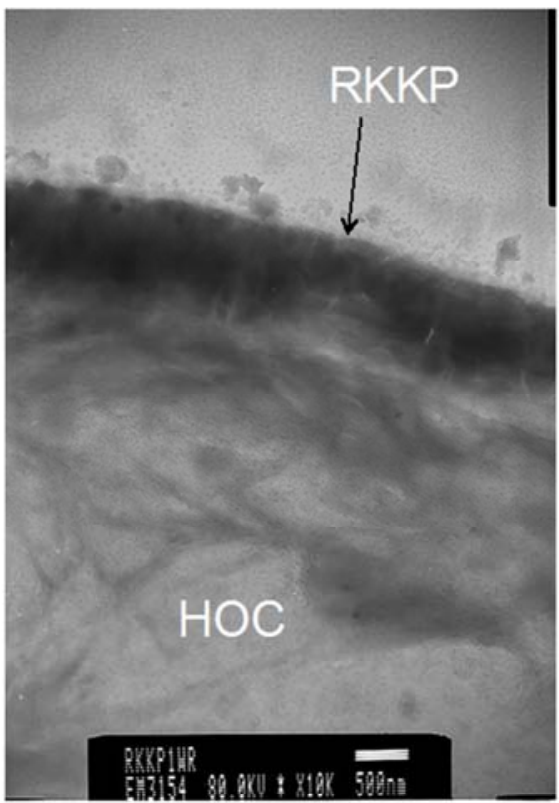

$\times 10 \mathrm{~K}$

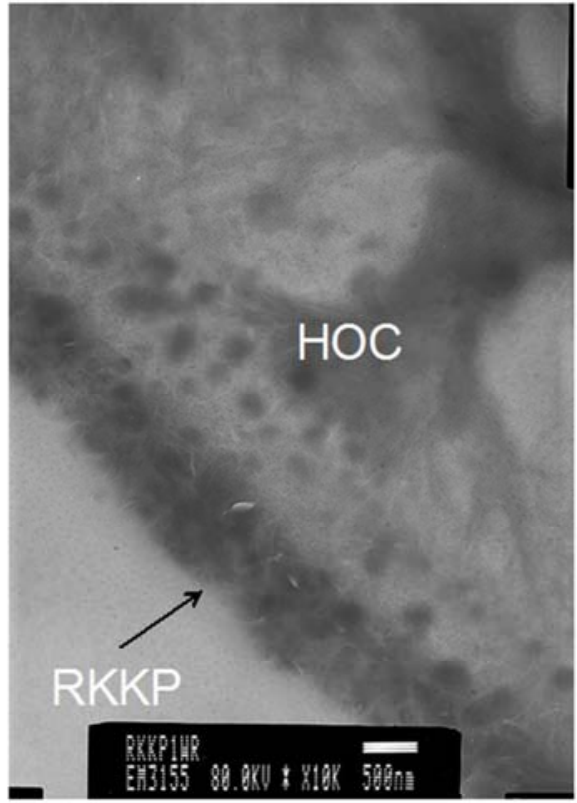

$\times 10 \mathrm{~K}$

RKKP (1 W)

Figure 6. TEM photographs of RKKP at 1 week after incubation. RKKP is bound directly to the collagen fibers of the HOC. The findings were completely different to those observed on RBP1 and RBP2. 


\section{Discussion}

Between 1970 and 1980, various biomaterials were developed and studied. These developments have led to the biomaterials that are currently in use. In the dental field, mainly, titanium alloy has been utilized clinically in dental implants. Hydroxyapatite is used as a material to coat titanium and to fill bone defects. Although bioglasses were applied clinically in the past for certain period of time, at present, they are not actively used due to their fragility. However, studies of bioglasses have continued from Hench' glass to A/W glass, AP40, RKKP, RBP1and RBP2.

In the present study, the interface between HOCs and Falcon or M-Ti, and RBP1, RBP 2, and RKKP showed large differences. In Falcon and M-Ti, a double-layered structure was observed between the cells and the sample. An extremely thin, dense layer was observed on the sample side. This layer was suggested to be some type of extracellular matrix related to cell adhesion. Moreover, we believe that this homogeneous layer produced adhesive proteins at the site of cell adhesion. We do not yet fully understand the clinical significance or role of this layer, but the cellular matrix that is produced apparently promotes the adhesion of cells to inert materials, similar to that which occurs with biomaterials. In contrast, a similar layer was not observed on the sample side of RBP1 or RBP2. These findings are suggested to indicate differences between the characteristics of bioinert and bioactive materials. Hence, the adhesion of cells to bioinert biomaterials (Falcon M-Ti) requires the presence of some kind of adhesive protein. On the other hand, the adhesion of cells to bioactive biomaterials (RKKP, RBP1, RBP2) might not require an adhesive protein because cells can adhere directly and/or intervening gel-like layer of bioglass to bioactive biomaterial.

As for RBP1 and RBP2, a structured layer (Figure 3, 4, 5) was found between the cells and the bioglass. There are three possible interpretations of this layer: it may represent a cellular matrix produced by adhesion with RBP1 or RBP2; it could be a gel-like substance; or it could represent other components. Seven studies have used TEM to morphologically examine the interface between bone cells and tissues and bioglass under different experimental conditions [5, 14-19]. Hench (Bioglass ${ }^{\circledR}$ ) is the only material that is reported to have a similar layer between the bone cells and bioglass. Oguchi et al. reported the biological evaluation of RBP1, 2. In 2014, we demonstrated the biological responses of the interface between new bioglasses (RKKP, RBP1, RBP2) and HGE cells using TEM. As a result, only RKKP was found to directly bond to cells without an intervening layer. On the other hand, in RBP1 and 2, we observed a gel-like layer at the interface on TEM photographs [15]. The gel-like structure of $\mathrm{RBP}$ suggested that the presence of $\mathrm{ZnO}$ in both RBP1 and RBP2 probably caused the formation of the gel layer. $\mathrm{Zn}_{2}{ }^{+}$ ions are mostly substituted by $\mathrm{Ca}_{2}{ }^{+}$or $\mathrm{Mg}_{2}^{+}$(as network modifiers); however, with a distorted ligand field, they may even be substituted by $\mathrm{Si}_{4}^{+}$(with a tetrahedral ligand field). Thus, $\mathrm{Zn}_{2}{ }^{+}$ions in Bioglass ${ }^{\circledR}(\mathrm{RBP} 1$ and $\mathrm{RBP} 2)$ play an important role in molecular and ionic stability. When $\mathrm{ZnO}$ comes into contact with a small amount of water it assumes an anionic character as zincyl ions and behaves as a gel. Experimentally, $\mathrm{Zn}_{2}{ }^{+}$is useful for controlling the solubility of glass systems and stabilizes the structure of Bioglass ${ }^{\circledR}[20]$. The present experiment showed no clear biological differences between RBP1 and RBP2 (despite the presence of some different components). Our results suggest that the action of $\mathrm{ZnO}$, which is contained in both RBP1 and RBP2, had a stronger impact on cellular differentiation than any of the other components. The structured layer seen on the cell side of the surface of RBP2 (Figure 5) might represent different crystalline phases due to the presence of not only $\mathrm{Zn}^{2+}$, but also $\mathrm{Sr}^{2+}$ (acting as a promoter). Furthermore, there were no biological differences between RBP1 and RBP2 in comparison to AP40. We conclude that $\mathrm{ZnO}$ might have a different effect on the biological response of cells in comparison to the other components ( $\mathrm{Nb}$ and $\mathrm{Sr})$.

Krajewski et al. [20] compared the behavior of two bioactive silica-phosphate glasses, AP40 and RKKP, in a simulated biological environment. As a result, IR and EDX analyses showed that the deposits formed on both glasses were composed of a calcium-deficient carbonate-apatite; however, the layer formed on the RKKP glass was found to be slightly more calcium-deficient and thinner. An EDX analysis evidenced the presence of a small percentage of F-ions, but only in the layers formed on the RKKP. It is well known that fluorine ions stabilize the apatitic lattice [21] and a small amount stimulates bone reconstruction (very small quantities of F-ions enhance osteoblast proliferation) [22]. Thus, the relationship between bone and bioglass, including the in vivo and in vitro mechanisms, has been investigated in detail. Bosetti et al. [23] reported that fibroblasts and osteoblast-like cells cultured on RKKP and AP40-coated zirconia showed a higher proliferation rate, leading to confluent cultures with a higher cell density and a generally better expression of osteoblast alkaline phosphatase activity in comparison to zirconia substrate. In conclusion, these results indicate that the surface chemical characteristics of the two glass coating AP40 and RKKP (which show similar properties), substantially enhance zirconia integration with bone cells (at least in vitro). Fini et al. [9] compared HA, Ti-6Al-4V, Zirconia, Alumina, AP40, RKKP in a histomorphometric study using a rat model of osteopenia. The study did not identify which materials gave the best results; however, they proved the affinity of RKKP for the osteopenia.

On the other hand, only RKKP bound directly to the cells without an intervening layer. These are the first TEM photographs in the world to show the relationship between bone cells and RKKP. RKKP glass ceramics containing minor amounts of apatite crystals ( $8 \%$ ) in a glassy matrix show good protein binding capacities [8, 24]. Its non-isothermal crystallization behavior was studied [12]. Small amounts of $\mathrm{La}_{2} \mathrm{O}_{3}$ and $\mathrm{Ta}_{2} \mathrm{O}_{5}$ were added to furnish a possible nucleus of deposition for the ions involved in bone formation. It was demonstrated that the presence of these oxides could modify 
the surface properties of the glass and influence the protein absorption kinetics in vitro [23]. Many researchers have also reported on the properties and biocompatibility to bone tissue in vivo and in vitro [4, 23-29]. Nicoli et al. (25) investigated the biocompatibility and osteointegration of zirconia $\left(\mathrm{ZrO}^{2}\right)$, either coated with RKKP bioglaze or uncoated, in vitro and in vivo. Histomorphometry demonstrated that at 30 days, the affinity index Furthermore, Stanic et al. [26] evaluated the osteointegration of yttria stabilized tetragonal zirconia (YSTZ), either coated with RKKP or uncoated in an animal model (Spraque Dawley rats) for 30 and 60 days. An in vivo histomorphometric evaluation revealed that at 30 days, the RKKP-coated YSTZ implants showed a significantly higher affinity index than the uncoated YSTZ implants. At 60 days, the coated implants behaved better than the controls, but the difference was not statistically significant. Bosetti et al. [23] reported that fibroblasts and osteoblast-like cells cultured on RKKP and AP40-coated zirconia showed a higher proliferation rate, leading to confluent cultures with a higher cell density and a generally better expression of osteoblast alkaline phosphatase activity in comparison to zirconia substrate. In conclusion, these results indicate that the surface chemical characteristics of the two glass coatings, AP40 and RKKP (which are highly similar) substantially enhance zirconia integration with bone cells (at least in vitro). Fini et al. [9] histomorphometrically compared HA, Ti-6Al-4V, Zirconia, Alumina, AP40, RKKP in a rat model of osteopenia. Although the study did not identify which material provided the best results, it proved the affinity of RKKP for osteopenia.

Our morphological study using TEM supported the findings of the above-mentioned reports. Furthermore, we concluded that RKKP bonded directly to HOCs and HGEs, indicating that it may be an extremely useful bioactive glass.

\section{Conclusion}

The interfaces between the bioactive materials and the bio-inactive material showed completely different phenomena. Furthermore, even the same bioactive materials showed different patterns because of their different compositions. RBP1 and RBP2 showed a gel-like layer. $\mathrm{M}-\mathrm{Ti}$ showed a non-structured homogeneous layer. In contrast, Falcon bonded directly to an extremely thin, dense layer, which was suspected to be some type of adhesive protein from the cells. RKKP bonded to collagen fibers with no intervening layer.

Based on these results, we conclude that RKKP might be the best biomaterial because the bone cells were observed to bind directly to RKKP without an intervening layer.

\section{Acknowledgements}

This study was confirmed by the ethics committee of Tsurumi University School of Medicine (No 420).

The authors declare no conflicts of interest in association with the present study.

\section{References}

[1] PT Proussaefs, DN Takakis, J Lozada, N Caplanis, MD Rohreri (2000) Histological evaluation of hydroxyapatite-coated root-form implants retrieved after 7 years in function. A case report. International of Journal Oral Maxillofacial Implants 15, 438-443.

[2] A Eliasson, T Eliksson, A Johansson, A Wennerberg. (2006) Fixed partial prostheses supported by 2 or 3 implants: A retrospective study up to 18 years. International of Journal Oral Maxillofacial Implants 21, 567-574.

[3] W Schulte, B D'Hoedt, D Axmann, R Romeg. (1992) 15 Jahre Tuebinger Implantat und seine Weiterentwicklung zum Frialit 2(R)-System. Zahnaerztlich Implantology 8, 77-96.

[4] LL Hench, RJ Splinter, TK Greenlee, Cl Allen. (1971) Bonding mechanisms at the interface of ceramic prosthetic materials. Journal of Biomedical Material Research 2, 117-141

[5] LL Hench, HA Paschell. (1971) Histochemical responses at a biomaterial's interface. Journal of Biomedical Material Research 5, 49-64.

[6] LL Hench. The story of Bioglass ${ }^{\circledR}$. (2006) Journal of Material Science: Material Medicine 17, 967-978.

[7] T Yamamuro. (1990) “A/W Glass-ceramic: Clinical applications, in handbook of bioactive ceramics: 1: Bioactive glass and glass ceramics." Edited by T Yamamuro, LL Hench (CRC press Boca Ranton, Florida) 335-342.

[8] A Krajewski, R Malavolti, A Piancastelli. (1996) Albumin adhesion on some biological and non-biological glasses and connection with their Z-potentials. Biomaterials 17, 53-60.

[9] M Fini, G Giavaresi, N Nicoli Aldini, P Torricelli, G Morreone, GA Guzzardella, R Giadino, A Krajewski, A Ravaglioli, MM Belmonte, AD Benedittid, G Biagini. (2000) The effect of osteopenia on the osteointegration of different biomaterials: histomorphometric study in rats. Journal of Material Science Material in Medicine 11, 579-585.

[10] P Torricelli, E Verne, BC Vital, H Appendino, A Rustichelli, A Krajewski, A Ravaglioli, G Pierni, M Fini, G Giavaresi, R Giardino. (2001) Biological glass coating on ceramic materials: in vitro evaluation using primary osteoblast culture from healthy and osteopenic in Rat. Biomaterials; 22, 2535-2543.

[11] A Krajewski, A Ravaglioli, A Tinti, P Tadddei, M Mazzocchi, R Martinetti, D Fagnano, M Fini. (2005) Comparison between the in vitro surface transformation of AP40 and RKKP bioactive glasses. Journal of Biomedical Material Research 16, 119-128.

[12] F Branda, G Luciani, B Silvesti, M Colella, A Costatini, A Ravaglioli, A Piancastli. (2004) Influence of $\mathrm{Ta}_{2} \mathrm{O}_{5}$ addition on the crystallization behavior of a bioactive glass (AP40). Physical Chemical Glasses 45, 343-347.

[13] A Ravaglioli, A Krajewski, A Piancastelli, G Baldi, A Barzanti, A Antoniini, M Mazzocchi. (2007) A comparison among some different doped bioactive glasses tested in vitro for tissue engineering. Ceramics, Cells and Tissues 11, 258-262.

[14] H Oguchi, Y Karube, K Matsumoto, M Morito. (2008) Response of Human Gingival Epithelial Cells (HGE-15 cells) to Bioactive Glass RKKP (surfaces as machined). Prosthodontic Research \& Practice 17, 5-11. 
[15] H Oguchi, Y Karube, Y Raita, T Ohshima, N Maeda. (2014) Responses of human epithelial cells (HGE-15 cells) to RBP1 and RBP2 bioactive glass as evaluated by scanning and transmission electron microscopy. Tsurumi University Dental Journal 40, 7-17.

[16] U Gross, J Brandes, V Strunz, J Sola. (1981) The ultrastructure of interface between a glass ceramics and bone. Journal of Biomedical Material Research. 15, 291-305.

[17] U Gross, V Strunz. (1985) The interface of various glasses and glass ceramics with a bony implantation bed. Journal of Biomedical Material Research. 19, 251-271.

[18] M Neo, S Kotani, T Nakamura, T Yamamuro. (1992) A comparative study of ultrastructures of the interfaces between four kinds of the surface-active ceramics and bone. Journal of Biomedical Material Research. 26, 1419-1432.

[19] MA Lingarten, CH Lai. (1975) Ultrastructure of the intact interface between an endosseous epoxy resin dental implant and host tissues. Journal of Biological Buccal. 3, 13-28.

[20] A Krajewski, A Ravaglioli, A Tinti. (2005) Comparison between the in vitro surface transformations of AP40 and RKKP bioactive glasses. Journal of Material Science Material medicine 16, 119-128.

[21] MI Key, RA Young, AS Posner. (1964) Crystal structure of hydroxyapatite. Nature 204, 1050-1055.

[22] LKH William, DJ Baylink. (1998) Molecular mechanism of action of fluoride on bone cells. Journal of Bone Mineral Research 13, 1660-1667.
[23] A Rosengren, S Oscarsson, M Mazzocchi, A Krajewski, A Ravaglioli. (2003) Protein absorption onto two bioactive glass-ceramics. Biomaterials 24, 147-155.

[24] M Boseti, E Verne, M Ferraris, A Ravaglioli, M Cannas. (2001) In vitro characterization of zirconia coated by bioactive glass. Biomaterials 22, 987-994.

[25] N Nicoli, M Fini, P Giacaresi, P Torricelli, L Martini, R Giadino, A Krajewski, A Ravaglioli, M Mazzochi, B Dubini, MG Ponzi, F Rustichelli, V Stanic. (2002) Improvement in zirconia osseointegration by means of a biological glass coating: An vitro and in vivo investigation. Journal of Biomedical Material Research 61, 282-289.

[26] V Stanic, AN Nicoli, M Fini, G Giararesi, R Giardino, A Krajewski, A Ravaglioli, M Mazzocchi, B Dubini, MG Ponzi, F Restichelli. (2002) Osteointegration of bioactive glass-coated zirconia in healthy bone: an in vivo evaluation. Biomaterials 23, 3833-3841.

[27] BM Mattioli, A Benedittis, RAA Muzzarelli. (1998) Bioactivity modulation of bioactive materials in view of their application in osteoporotic patients. Journal of Material Science: Material Medicine 9, 485-492.

[28] E Verne, M Ferraris, A Ventrella, L Oaraxxhini, A Krajewski, A Ravaglioli. (1998) Sintering and plasma spray deposition of bioactive glass-matrix composites for medical applications. Journal of European Ceramic Sociality 18, 363-372.

[29] M Ferraris, E Verne, P Appendino, C Moisescu, A Krajewski, A Ravaglioli, A Piancastelli. (2000) Coatings on zirconia for medical applications. Biomaterials 21, 765-773. 